\title{
Performance of Dynamic Priority Based Interference Aware Channel Allocation in Wireless Mesh Networks for Energy Conservation
}

\author{
Jasmine David. D, Jegathesan V, Roopa Jayasingh.J
}

\begin{abstract}
The Dynamic Wireless Mesh Network (DWMN) infrastructure is a pair or multiple dynamic nodes with networking capability to communicate with one another utilising Sink Nodes (SN). Due to its mobile nature, it is termed as Dynamic in nature. SN traverses over the predefined path over the Wireless Mesh Network. As the nodes engage into mobility, the neighbourhood table should be updated at a minimum rate of once every five seconds. The alarming fact in WMN is, Energy to transmit a bit is equivalent to computing hundreds of instructions at that instant. Hence there is a need to concentrate on energy dimension of DWMN. A node loses certain amount of energy while transmitting and receiving the packet, hence there is a minor decline in the initial energy of the node. The existing value of energy at a moment after transmitting and receiving the packet is coined as Residual Energy. The energy consumed by the node for transmitting and receiving the packet over the particular time frame is coined as Average Energy. Network Simulator v.2 tool has been utilised to simulate network creation with multiple mobile nodes for packet transmission or reception and packet drop conditions due to interference. Bandwidth Reservation (BR) for energy analysis is done by both Priority Based Interference Aware Bandwidth Reservation (PBIABR) and Interference Aware Bandwidth Reservation (IABR) for many flowing rates under dynamic scenario. Channel Priority plays a vital role to opt the channel which posses less interference for efficient bandwidth reservation for PBIABR. The opted channel will have the minimal channel interference effect. IABR posses the controllability character for data flow to establish end-to-end communication over Multi-Radio Multi-Channel (MRMC) - Wireless Mesh Network. This research paper focuses on deep analysis of Residual Energy (joule) compared with Interval (sec) and Average Energy (joule) compared with Interval (sec) under dynamic scenario for multiple flow rates by implementing PBIABR and IABR criteria.
\end{abstract}

Keywords : Dynamic Wireless Mesh Network, Residual Energy, Sink Nodes, Priority Based Interference Aware Bandwidth Reservation, Interference Aware Bandwidth Reservation

Revised Manuscript Received on November 08, 2019.

Correspondonce author ${ }^{*}$

Jasmine David , Asst.Prof, Department of ECE, Karunya Institute of Technology and Sciences, Coimbatore, India. Email: jasmine@karunya.edu

Jegathesan V, Associate Prof, Department of EEE, Karunya Institute of Technology and Sciences, Coimbatore, India. Email:jegathesan.v $@$ karunya.edu

Roopa Jayasingh.J ,Asst Prof, Department of ECE, Karunya Institute of Technology and Sciences, Coimbatore, India. Email: roopa@karunya.edu

\section{INTRODUCTION}

Wireless Mesh Network (WMN) is the trouble-free cost-reducing alternative for Communication Network Operators (CNO) to assure a high-speed connectivity and access to specific areas where the application of cellular Network is difficult. The interesting fact is that the WMN can broaden cellular network based femto-cell applied coverage areas with a back-haul internet dorsal and hence they can drive multiple user compatibility. Advanced invention in Wireless Mesh Network (WMN) brings the dream of connecting every object as a well formed connected world Mesh Nodes (MN) is a prime backbone for seamless data communication in WMN. Nodes behave as parent and child to its organised network i.e. it act as data provider and data receiver in the network [1-2]. The organization in the WMN is decentralised in nature which enables fault tolerant and reliable data communication in the connected network. These advancement and flexibility in Wireless Mesh Network (WMN) enables the rural community which operates with the miniaturised network to connect with the fascinating Internet with the ease of affordability [3].

\section{A.Dynamic Wireless Mesh Networks (DWMN)}

Wireless Mesh Network (WMN), an advancement from ad-hoc network, is a alluring data communication network which encompasses radio nodes which are structured in a Mesh Topology (MT). Simplest form of MT is interconnection between at least three nodes for fault tolerance to each other. The Infrastructure Node (IN) like hub routers, switches and etc. are connected to as many nodes as possible in the network. The prime advantage of mesh network is that it can dynamically self-organise and self-configure, due to this functionality the installation over head is placated effectively. MN topology usually is in the form of conventional Star/Tree topologies, whereas the bridges or switches directly link to the other mesh network. Wireless Nodes (WN), are built with programmed software that allows them to communicate with the wider network. Data are hopped from one mesh to another mesh via multiple nodes in-between them. During the course of data transfer via the mesh layout, the nodes share the network status thereby making them aware about the entire network. Nodes automatically finds the shortest path and safest path, hence it is coined as Dynamic Network Communication (DNC) in nature. Fig. 1. Portrays the evolution of Mesh Network 
(MN) from basic network topologies.

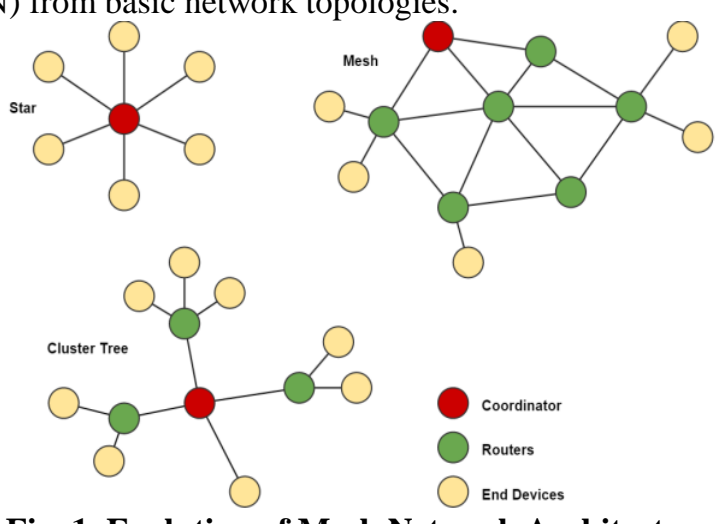

Fig. 1. Evolution of Mesh Network Architecture.

In Star Network, the end devices connect to the centralised coordinator for data communication directly. In Cluster Network, multiple end devices which are connected to the router are interconnected to the coordinator effectively. Mesh Network communicates with the other mesh network by establishing the connection between two co-ordinator nodes of the mesh network. During data communication coordinator nodes shares the entire information of the mesh network with one another to establish the seamless communication

\section{B.Dynamic Multi Radio Mesh Network (DMRMN)}

Dynamic Multi Radio Mesh Network (DMRMN) indicates the exclusive couplet of devoted radios devices on every terminal end of the connection. There exist an exclusive frequency used for every cable-less hop and thus a committed Carrier Sense Multiple Access (CSMA) collision domain. This establishes the proper mesh link to achieve utmost network performance devoid of channel bandwidth compramization in the cable-less mesh and with nil additive latency. Owing to this functionality voice and video applications work same as on a wired Ethernet network. In DMRMN, a node will be devoted to one of the radios, to act as a station, and the other to connect neighbour node Access Point (AP) in the mesh network [4].

\section{C.Channel Allocation in Dynamic Wireless Mesh Network (DWMN)}

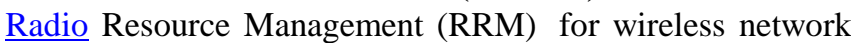
and cellular network for Dynamic Wireless Mesh Network communication, channel allocation schemes are compulsory to allot bandwidth and Radio Communication Channel (RCC) for base stations, access points and to all the endpoint equipment [5].

\section{$D$. Network Interference (NI) in Channel}

Interference which is additive factors in the channel which alters or disrupts a signal as it travels among a source module and receiver module. It makes sense as an additive undesired signal to desired signal. Few interference in the channel are as listed Intersymbol interference (ISI), CoChannel Interference (CCI), Inter Carrier Interference (ICI), which is due to Doppler shift oriented channel interference in Orthogonal Frequency Division Multiplexing ( $\underline{\text { OFDM }}$ ) signal modulation which is a multi-tone modulation and Common-mode interference (CMI). Interference is not always distinguished from noise, for example white noise. Radio Communication aims at plummeting and controlling the co-channel and adjacent-channel interference. Channel Interference affects the successful rate of data communication [6].

\section{RESIDUAL ENERGY IN WIRELESS MESH NETWORK (WMN).}

A Wireless Node loses a certain quantity of energy for each transmitted and received data packets. When this continues through the course of data communication, the value of Initial Energy ( ) in a node gets diminished by certain quantity. The present energy level of Wireless Node (WN) after data transmission or reception for routing purpose is the residual energy.

In multi-datagram-hop DWMN, the retaining Residual Energy (RE) of the node at the end of a time frame $(t+1)$ which depends on the consumed and replenished energy for data transmission and reception during this time frame. $\mathrm{Bi}(\mathrm{t})$ and $\mathrm{Bi}(\mathrm{t}+1)$ as the keeping hold the residual energy of specific node $i$ at the dawn and the commencement of time frame $t$ and $(t+1)$. The component, $B i(t+1)$ is thus the retaining residual energy of node $i$ at the end of time frame $t$, correspondingly. Ei(t) symbolize the analogous replenished nodal energy which is exceedingly connection to geographical location and other prevailing natural factors. Owing to nodal energy stage of a mesh network nodal energy source is constrained between Bout and Bmax, the retaining residual energy of the node $i$ at the termination of time slot $t$ is $\mathrm{Bi}(\mathrm{t}+1)=\min [\operatorname{Bmax}, \max [\operatorname{Bout} \mathrm{Bi}(\mathrm{t})+\mathrm{Ri}(\mathrm{t})-\mathrm{Bi}(\mathrm{t})]\}-1$

Where, $\operatorname{Ri}(\mathrm{t})=\operatorname{ri}(\mathrm{t}) \cdot \mathrm{T}$, and $\operatorname{ri}(\mathrm{t})$ is where the replenishment rate of energy that change in a dynamical manner.

\section{SIMULATION SCHEMA FOR DWMN}

Each node in WMN is assigned to work with five Radio Interfaces. One dedicated radio interfaces is assigned for Control Interfaces (CI) operation and the rest four radio interfaces are for Data Interfaces (DI). Both the physical hardware and Media Access Control (MAC) level protocol is reduced as per IEEE $802.1 \mathrm{~b} / \mathrm{g}$ protocol. Table 1 , highlights various basic channel parameters for WMN creation.

TABLE 1. Simulation Schema Parameter for DWMN

\begin{tabular}{|c|c|}
\hline Parameter & Value \\
\hline Channel Capacity & $11 \mathrm{Mpbs}$ \\
\hline Interference Range & $200 \mathrm{~m}$ \\
\hline Transmission Range & $100 \mathrm{~m}$ \\
\hline Channel Assignment & Non Overlapping : 3 \\
\cline { 2 - 2 } & Partially Overlapping : 8 \\
\hline
\end{tabular}

For the purpose of Interference Aware Bandwidth Reservation (IABR) and also Priority based Interference Bandwidth Reservation (PBIABR) learning, the dynamic network topology of 100 wireless routers spread over the wide span of about $1000 \mathrm{~m}$ x $1000 \mathrm{~m}$ area. Successive multicast session is modelled by designing newly originating multicast tree the hybrid network topology. Multicast Tree (Tn) evolving to the newly evolved multicast session which is formed by the random choosing of 10 leaf nodes. Based on the reduced Heuristic based Algorithm the used channel of every node is decided on the multicast tree. The Parent Node (PN) of the multicast tree pushes 100 units of multicast data packets for every second. The dimensional weight of every packet is prealloted to 1024 bytes. Upon receiving the multi casted datagram packet from the various $\mathrm{PN}$, the node on every tree uses its channel to distribute the packet to its identifiable Child Nodes $(\mathrm{CN})$.

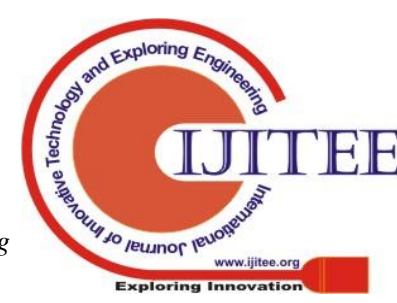


The pointed out data's which are made as a setup which uses Matrix Laboratory (MATLAB). That simulating environment informations which has been arrived to NS2 simulator inorder to pick up the trace for every packet transmission under analysis.

\section{BANDWIDTH RESERVATION TECHNIQUES}

A.Interference Aware Bandwidth Reservation - IABR. Interference Aware Bandwidth Reservation (IABR) schemes are utilised for the single flow of reservation for various bandwidth allocation in the Wireless Mesh Network (WMN). IABR propose a model-oriented methodology which would smartly capture the effect of intra and inter flow able interference and thus provide appropriate estimating of the bandwidth [11-12]. This methodology concentrates towards MRMC networks and utilises link-diversity (many links between neighboring nodes are established) [13-14].

B.Priority Based Interference Aware Bandwidth Reservation - PBIABR.

PBIABR based bandwidth reservation methodology which shows a scattered and polynomial-oriented time heuristic CA to placate interference along the channel in Wireless Mesh Networks [15-16]. This methodology directs the channel assignmening difficulty by taking the consideration of interference in WMNs, and then produces the best possible outcome to the CA hitch. PBIABR algorithm placate the priority oriented aware interference effects for currently existing multicast session.

Contrasting to the previous CA algorithms, the current method uses all the achievable channels of the Wireless Mesh Network to achieve CA which are most CA algorithms in WMNs which use only nil-overlappable channels. Fig. 3., shows the functional procedure found in Priority Based Interference Aware Bandwidth Reservation (PBIABR).

The prime factor for the sounding performance in PBIABR is the additional functional block i.e. Inter Tree Interference, Accumulated Average [17-18].

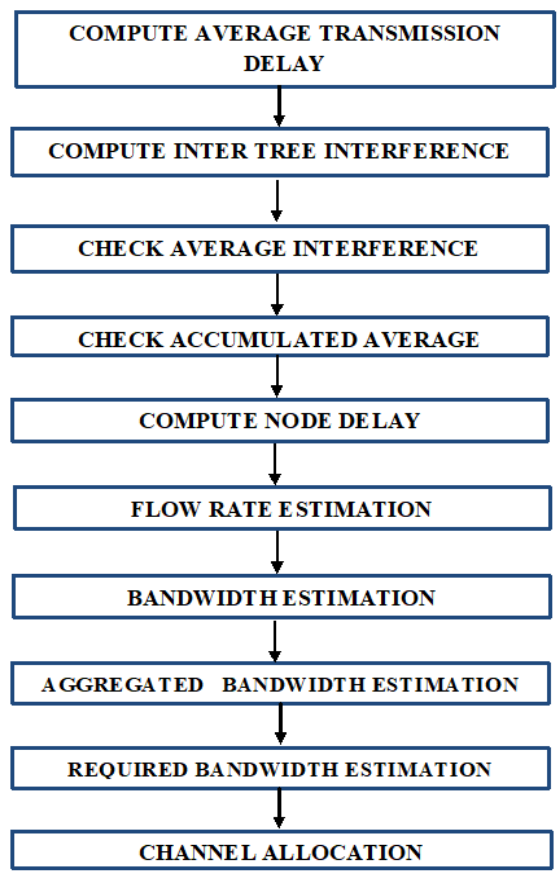

Fig. 3. Priority Based Interference Aware Bandwidth Reservation - PBIABR methodology.

\section{RESIDUAL ENERGY ANALYSIS UNDER IABR AND PBIABR IN DYNAMIC MODE.}

Residual Energy (RE) is the available energy after the node functions and exhaust over the data communication. The prime motive behind the calculation of RE is that it should be above the mean average energy of the node for promise the stable data communication [19]. The amount of retained residual energy impacts the bandwidth of the channel as the nodes are associated for data communication [20]. Under our prime research analysis of the average calculated RE of collective 100 nodes over the WMN data communication. Interference has been added over the data communication channel. For each analysis penta dissimilar flows for IABR and PBIABR have been analysed.

From the simulated statistical results the analytical graph have been plotted with residual energy which is is usually measured in joules found along the vertical axis and the time interval which is also calibrated in seconds found along the horizontal axis. Stepping range of 0.05 joules for the effective $\mathrm{RE}$ analysis has been set for precise understanding. Stepping range of 10 seconds, along the horizontal axis has been set for time frame to hold a accurate control over the minute variation over the period of network. Fig. 4., depicts the analytical 2-Dimensional plot of a Residual Energy vs Interval under dynamic scenario. To highlight the difference of multiple flow rates in the graph, unique colours have been utilized for variation understanding.

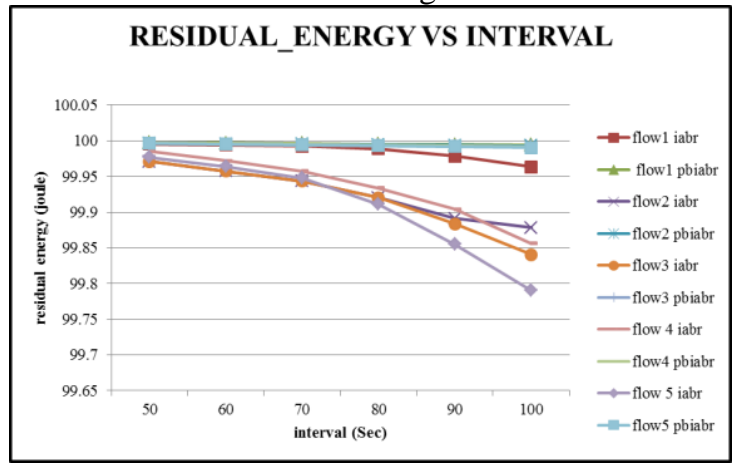

Fig. 4. Residual Energy (joule) vs Interval (Seconds) under IABR and PBIABR.

Inference from the analytical graph is that the till the time interval of 80 units the flow follows the streamlined path. Beyond this the variation are evident enough to prove that the PBIABR is best technique to retain the residual energy of the prevailing nodes in the mesh network. Drastic variation of performance is evident for the flow 5 which traces along the residual energy range, between, 99.95 - 100 joule. Flow 5 is taken as a evident sample to showcase the effect of bandwidth reservation and its effect on residual energy for effective analysis. Generally, when time interval increases the amount of retained RE tends to decrease due to loss in channel medium and in mesh nodes. Flow 5, under IABR implementation drops to 99.79 joules when the interval approaches to 100 seconds. Flow 5, under PBIABR implementation maintain the standard flow of energy retain of 99.99 throughout the interval range. This analytical comparison is the bright evident 


\section{Performance of Dynamic Priority Based Interference Aware Channel Allocation in Wireless Mesh Networks for Energy Conservation}

that PBIABR is best suited for bandwidth reservation in WMN.

\section{AVERAGE ENERGY ANALYSIS UNDER IABR AND PBIABR IN DYNAMIC MODE.}

Average Energy (AE), is the amount of consumed energy over the time frame by wireless nodal components in mesh network. To establish a well performing network, the consumed average energy should be made minimal. By analysing $\mathrm{AE}$, the amount of energy spent for data communication for packet transmission over the connected network. Utilised AE, by the 100 nodes have been specified as per the parameter layout for effective analysis. Fig. 5., portrays the graphical analysis of Average Energy which is calculated in joule versus Interval which is also calculated in Seconds under IABR and PBIABR.

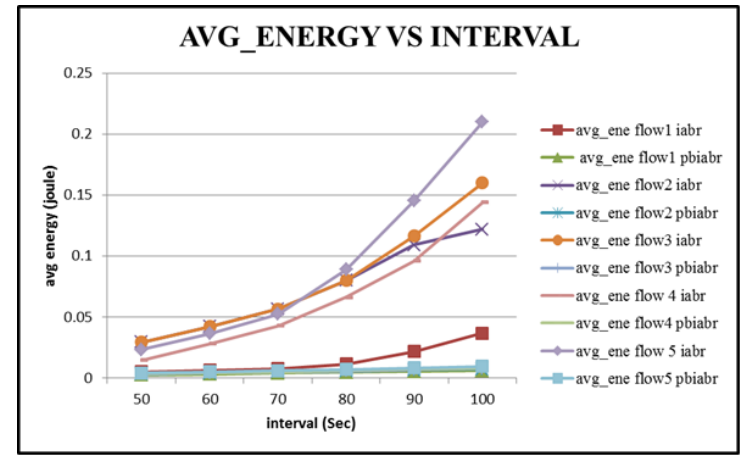

Fig. 5. Average Energy (joule) vs Interval (Seconds) under IABR and PBIABR.

Analytical interpretation from the graphical representation of flow 5 under two criteria, can be the bright evident for the level of energy utilised under IABR and PBIABR implementation. Flow 5 under IABR, raises exponentially from 0.02324 to 0.20165 joules over the time frame of 50 to 100 seconds with the stepping range of 50 units. Flow 5 under PBIABR, consumes a minimal stable consumption of energy with the average of 0.00591 over the entire time frame. Thus, Average Energy, is utilised efficiently for PBIABR implemented communication which allocates the prime bandwidth resource under priority

\section{CONCLUSION}

Energy is the prime factor which plays the vital role in entire Wired and Wireless Communication Spectrum. Channel Assignment (CA) for best bandwidth reservation is profoundly needed in mesh network. In analogy to existing algorithm, the proposed CA technique exploits partially overlapping channel along with the nil-overlapping channels. The retaining residual energy when, PBIABR reservation technique is applied along the nodes is much higher than the channel where only IABR is applied. PBIABR overrides the existing IABR by factor of priority based reservation for channel allocation. Simulation results highlights the attained energy efficiency by attaining maximum residual energy retain factor for each flow. Average Energy holds the minimal value while implementing PBIABR technique for reservation of bandwidth as required for effective cable-less radio communication. Thus, the implementation of PBIABR for reservation schema is best suited for maximum energy utilization along the mesh nodes.

\section{REFERENCES}

1. I. F. Akyildiz and X. Wang, "A survey on wireless mesh networks," IEEE Commun. Mag., vol. 43, no. 9, pp. 23-30, Sept. 2005.

2. D. Benyamina, A. Hafid, and M. Gendreau, "Wireless mesh networks design-a survey," IEEE Commun. Surveys \& Tutorials, vol. 14, no. 2 pp. 299-310, 2nd Quarter 2012.

3. Mathonsi, Topside E, and Okuthe P. Kogeda. "Enhanced Bandwidth Sharing Scheme for Small and Medium Enterprises." Proceedings of the World Congress on Engineering and Computer Science. Vol. 2. 2014.

4. A. P. Subramanian, H. Gupta, S. R. Das, and J. Cao, "Minimum interference channel assignment in multiradio wireless mesh networks," IEEE Trans. Mobile Comput., vol. 7, no. 12, pp. 1459-1473, Dec. 2008.

5. A. Capone, G. Carello, I. Filippini, S. Gualandi, and F. Malucelli, "Routing, scheduling and channel assignment in wireless mesh networks: Optimization models and algorithms," Ad Hoc Networks, vol. 8, no. 6, pp. 545-563, Aug. 2010.

6. D. Passos and C. V. N. Albuquerque, "A joint approach to routing metrics and rate adaptation in wireless mesh networks," IEEE/ACM Trans. Networking, vol. 20, no. 4, pp. 999-1009, Aug. 2012.

7. D. Gupta, P. Mohapatra, C. Chuah, Seeker "A Bandwidth-Based Association Control Framework For Wireless Mesh Networks", Wireless Networks (2011).

8. Ko, Bong-Jun, et al. "Distributed Channel Assignment in multi-radio 802.11 mesh networks." Wireless Communications and Networking Conference, WCNC (2007).

9. Tu, Xiaoyu, Hai Wang and Zhimin Li. "Channel Vector: An overhead reduced broadcast in Multichannel Wireless Mesh Networks. "Communications (ICC), 2015 IEEE International Conference on. IEEE, 2015.

10. Bakhshi, Bahador, SiavashKhorsandi, and Antonio Capone. "On-line joint QoS routing and channel assignment in multi-channel multi-radio wireless mesh networks." Computer Communications 34.11, (2011).

11. Kaan Bur and CemErsoy, "Ad hoc quality of service multicast routing", Computer Communications, Volume 29, Issue 1, 2005.

12. Bosheng Zhou, Alan Marshall and Tsung-Han Lee, "A CrossLayer Architecture for DiffServ in Mobile Ad-hoc Networks", International Conference on Wireless Networks, Communications and Mobile Computing, WIRELESSCOM, 2005.

13. Bo Wang and Matt Mutka, "QoS-aware fair rate allocation in wireless mesh networks", Elsevier, Computer Communications 31, 2008

14. Gahng-SeopAhn, Andrew T. Campbell, AndrasVeres and Li-Hsiang Sun, "SWAN: Service Differentiation in Stateless Wireless Ad Hoc Networks", In the Proceedings of the 21st Annual Joint Conference of the IEEE Computer and Communications Societies, INFOCOM, 2002.

15. Ko, Bong-Jun, et al. "Distributed Channel Assignment in multi-radio 802.11 mesh networks." Wireless Communications and Networking Conference, WCNC (2007).

16. Y. Song, C. Zhang, and Y. Fang, "Joint channel and power allocation in wireless mesh networks: A game theoretical perspective," IEEE J. Select. Areas Commun., vol. 26, no. 7, pp. 1149-1159, Sept. 2008.

17. J. Luo, C. Rosenberg, and A. Girard, "Engineering wireless mesh networks: Joint scheduling, routing, power control, and rate adaptation," IEEE/ACM Trans. Networking, vol. 18, no. 5, pp. $1387-$ 1400, Oct. 2010

18. L. X. Cai, Y. Liu, T. H. Luan, X. S. Shen, J. W. Mark, and H. V. Poor, "Adaptive resource management in sustainable energy powered wireless mesh networks," in Proc. IEEE GLOBECOM'11, Houston, TX, USA, Dec. 5-9 2011, pp. 1-5.

19. L. Lin, N. B. Shroff, and R. Srikant, "Asymptotically optimal energy-aware routing for multihop wireless networks with renewable energy sources," IEEE/ACM Trans. Networking, vol. 15, no. 5, pp. 1021-1034, Oct. 2007

20. [20] J. Tang, G. Xue, and W. Zhang, "Maximum throughput and fair bandwidth allocation in multi-channel wireless mesh networks," in Proc. IEEE INFOCOM'06, Barcelona, Spain, Apr. 23-29 2006, pp. $1-10$. 


\section{AUTHORS PROFILE}

Mrs.Jasmine David.D did her UG \& PG in Karunya Institute of Technology and Sciences, Coimbatore,India. Her research is focused on Wireless Mesh Networks. She is currently working as Assistant Professor in Electronics and Communication Engineering, Karunya Institute of Technology and Sciences,Coimbatore, India.Her total teaching experience is 20 years. Her field of interest is Wireless Networks.

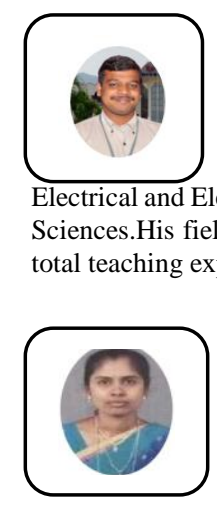

Dr.Jegathesan.V completed his UG \& PG in Karunya Institute of Technology and Sciences,Coimbatore, India.He completed his Ph.D degree in Anna University.Currently working as Assosiate Professor in Electronics Engineering, Karunya Institute of Technology and Sciences.His field of interest in Static Power Converts \& Inverters ,etc His total teaching experience is 20 years.

Dr. Roopa Jayasingh J She did her UG \& PG in Karunya University. She completed her PhD degree in Information and Communication Engineering under Anna University, Coimbatore. Her research focused on Biomedical Signal Processing. She is currently working as Assistant Professor in Electronics and Communication Engineering, Karunya University, Coimbatore. Her total teaching experience -13 Years 6 Months. Her field of Interest is Biomedical Engineering. 